\title{
Assessment of Helicobacter pylori eradication in patients on NSAID treatment
}

\author{
Harald E Vonkeman ${ }^{1 *}$, HTJ deLeest ${ }^{2 \dagger}$, MAFJ van deLaar ${ }^{1}$, J vanBaarlen ${ }^{3}$, KSS Steen ${ }^{2}$, WF Lems ${ }^{2}$, JWJ Bijlsma ${ }^{4}$, \\ EJ Kuipers ${ }^{5}$, HHML Houben ${ }^{6}$, M Janssen $^{7}$ and BAC Dijkmans ${ }^{2}$
}

\begin{abstract}
Background: In this post-hoc analysis of a randomized, double blind, placebo controlled trial, we measured the sensitivity and specificity of Helicobacter pylori lgG-antibody titer changes, hematoxylin and eosin (H\&E) stains, immunohistochemical $(\mathrm{IHC})$ stains and culture results in NSAID using patients, following H. pylori eradication therapy or placebo.
\end{abstract}

Methods: 347 NSAID using patients who were H. pylori positive on serological testing for H. pylori lgG-antibodies were randomized for $\mathrm{H}$. pylori eradication therapy or placebo. Three months after randomization, gastric mucosal biopsies were taken for $\mathrm{H}$. pylori culture and histological examination. At 3 and 12 months, blood samples were taken for repeated serological testing. The gold standard for $\mathrm{H}$. pylori infection was based on a positive culture or both a positive histological examination and a positive serological test. Sensitivity, specificity and receiver operating curves (ROC) were calculated.

Results: H. pylori eradication therapy was successful in $91 \%$ of patients. Culture provided an overall sensitivity of $82 \%$, and $73 \%$ after eradication, with a specificity of $100 \%$. Histological examination with either H\&E or IHC stains provided sensitivities and specificities between 93\% and 100\%. Adding IHC to H\&E stains did not improve these results. The ROC curve for percent change in $\mathrm{H}$. pylori lgG-antibody titers had good diagnostic power in identifying H. pylori negative patients, with an area under the ROC curve of $0.70(95 \% \mathrm{Cl} 0.59$ to $0.79, P=0.085)$ at 3 months and $0.83(95 \% \mathrm{Cl} 0.76$ to $0.89, P<0.0001)$ at 12 months. A cut-off point of at least $21 \%$ decrease in $\mathrm{H}$. pylori IgG-antibody titers at 3 months and $58 \%$ at 12 months provided a sensitivity of $64 \%$ and $87 \%$ and a specificity of $81 \%$ and $74 \%$ respectively, for successful eradication of $H$. pylori.

Conclusions: In NSAID using patients, following H. pylori eradication therapy or placebo, histological examination of gastric mucosal tissue biopsies provided good sensitivity and specificity ratios for evaluating success of $\mathrm{H}$. pylori eradication therapy. A percentual H. pylori lgG-antibody titer change has better sensitivity and specificity than an absolute titer change or a predefined $\mathrm{H}$. pylori lgG-antibody titer cut-off point for evaluating success of $\mathrm{H}$. pylori eradication therapy.

\section{Background}

Helicobacter pylori (H. pylori) infection has been shown to be related to the development of peptic ulcer disease, chronic gastritis, MALT lymphoma and gastric cancer [1-4]. Accurate diagnosis of $H$. pylori infection has clinical consequences as $H$. pylori eradication improves

\footnotetext{
* Correspondence: h.vonkeman@mst.nl

${ }^{\dagger}$ Equal contributors

'Arthritis Center Twente, Department of Rheumatology and Clinical Immunology, Medisch Spectrum Twente Hospital and University of Twente, P.O. Box 50.000, 7500 KA Enschede, The Netherlands

Full list of author information is available at the end of the article
}

outcome and recurrence of peptic ulcer disease. H. pylori infection can be detected using non-invasive tests such as serological tests, 13C-urea breath test and stool tests, and invasive tests requiring endoscopically obtained gastric mucosal tissue biopsies, such as tissue culture, examination of histological stains and the rapid urease test. Serological tests based on the detection of antibodies to $H$. pylori have been shown to have high sensitivity and are therefore useful in screening for $H$. pylori infection [5-7]. However, because serological tests merely detect an immune response, they do not discriminate between current or previous infection. H. pylori

\section{() Biomed Central}

(c) 2012 Vonkeman et al.; licensee BioMed Central Ltd. This is an Open Access article distributed under the terms of the Creative Commons Attribution License (http://creativecommons.org/licenses/by/2.0), which permits unrestricted use, distribution, and reproduction in any medium, provided the original work is properly cited. 
infection of the gastric mucosa causes a chronic local inflammatory cell infiltration, which in turn gives rise to a serological response, in which $H$. pylori specific antibodies are almost always detectable $[8,9]$. After successful $H$. pylori eradication therapy, the level of $H$. pylori specific antibodies decreases progressively over a period of several months, possibly parallel to the slowly healing inflammation of the gastric mucosa [10]. As a result, evaluating success of $H$. pylori eradication therapy using repeated serological tests has only been shown to be useful if a period of several months is maintained between tests [11-13].

Culture of $H$. pylori in biopsy specimens has very high specificity and allows testing for antibiotic susceptibility but has relatively low sensitivity and is labour-intensive [14]. Histological identification of $H$. pylori in biopsy specimens has long been considered to be the clinical standard for the diagnosis of $H$. pylori infection. A high density of $H$. pylori is readily apparent on routine hematoxylin and eosin ( $\mathrm{H} \& \mathrm{E})$ stains but detection of a lower density of bacteria may require additional staining techniques [15]. H. pylori is more easily visualised with immunohistochemical $H$. pylori antibody stains than with the standard H\&E staining. However, the use of immunohistochemical (IHC) stains adds time and expense to the diagnostic evaluation for $H$. pylori and is therefore not routinely performed.

The interaction between $H$. pylori infection and the use of non-steroidal anti-inflammatory drugs (NSAIDs) in the development of gastroduodenal ulcers remains unclear. In a meta-analysis of 16 endoscopic studies in NSAID users from various countries, uncomplicated gastric ulcer disease was twice as common in $H$. pylori positive patients as in $H$. pylori negative patients [16]. However, the rate of $H$. pylori infection in patients with NSAID associated gastric ulcers is significantly lower than in those with non-NSAID associated gastric ulcers [17]. Furthermore, while eradication of $H$. pylori infection in NSAID-naïve patients prior to NSAID therapy reduces the risk of ulcer development, it does not do so in current NSAID users [18-20]. This was also confirmed in a recent randomized, double blind, placebo controlled clinical trial, in which we found that eradication of $H$. pylori infection did not reduce the incidence of endoscopic gastroduodenal ulcers in $H$. pylori seropositive patients currently taking NSAIDs for rheumatic diseases [21].

H. pylori infection has been shown to induce cyclooxygenase (COX)-2 expression in the gastric mucosa, which persists during active $H$. pylori infection [22-25]. It has been suggested that COX-2 plays an immunosuppressive role in H. pylori gastritis [26]. Conversely, in H. pylori infected mice, NSAID treatment has been shown to significantly decrease the degree of gastric inflammation
[27]. It is therefore possible that in patients with $H$. pylori infection, concurrent NSAID treatment may affect levels of gastric inflammation and may consequently affect the serological response. While several studies have investigated the time course of $H$. pylori antibody titers after $H$. pylori eradication therapy, none have been conducted in NSAID users [9,11-13,28].

This study presents a post-hoc investigation into $H$. pylori IgG-antibody titer changes following $H$. pylori eradication therapy in NSAID users. In patients participating in the before mentioned $H$. pylori eradication in NSAID users trial, we measured $H$. pylori IgG-antibody titers and titer changes in order to diagnose successful $H$. pylori eradication [29]. We further compared H. pylori IgG-antibody titers, H\&E stains, IHC stains and $H$. pylori culture results in follow-up biopsies from $H$. pylori-positive NSAID-users randomized to eradication treatment or placebo, to determine the sensitivity and specificity of these different methods in NSAID users. Furthermore, we determined whether adding IHC stains to $H \& E$ stains improves the histological identification of $H$. pylori in these patients.

\section{Methods}

\section{Study design}

The methods of the primary randomized, double blind, placebo controlled clinical trial have been previously described in more detail ${ }^{21}$. Between May 2000 and June 2002, patients between the ages of 40 and 80 years with a rheumatic disease requiring NSAID treatment, were recruited and included in the study if tested positive for $H$. pylori on serological testing. During the study, no change in NSAID-therapy was permitted, but there was no restraint on other medication. Exclusion criteria were previous $H$. pylori eradication therapy and severe concomitant disease.

After stratification by concurrent use of gastroprotective agents (proton pump inhibitors, H2 receptor antagonists or misoprostol, but not prokinetics, or antacids), patients were randomly assigned to receive either $H$. pylori eradication therapy with omeprazole $20 \mathrm{mg}$, amoxycillin $1000 \mathrm{mg}$, and clarithromycin $500 \mathrm{mg}$ (OAC) twice daily for 7 days or placebo. Patients with an allergy for amoxycillin were randomized in a separate stratum to receive omeprazole $20 \mathrm{mg}$, metronidazol $500 \mathrm{mg}$ and clarithromycin $250 \mathrm{mg}$ (OMC) or placebo therapy twice daily for one week. Randomization to consecutive patient numbers was done in proportions of $1: 1$, in blocks of four from a computer-generated list. The study centers were provided with individually sealed packages containing the treatment for each patient. Each centre received entire blocks to be used sequentially. Rheumatologists were not practicing in more than one center. The study medication was given in a double blind, 
double dummy manner. Active and placebo preparations were identical in appearance. The employees of the VU University Medical Center pharmacy who packaged the medication only knew the assignment. It was disclosed to the treating physician only in case of emergency. All study personnel and participants were blinded to treatment assignment for the duration of the study.

After 3 months patients underwent gastroduodenal endoscopy, during which 4 antrum biopsies and 4 corpus biopsies were taken for culture and histological examination. After 3 and 12 months, blood samples were taken for repeated serological testing. Immunohistochemical staining was only available for a subset of patients recruited at the Medisch Spectrum Twente hospital in Enschede, the Netherlands. The study protocol was approved by the Institutional Ethical Review Board of all participating hospitals and all patients gave written informed consent.

\section{Serology}

Serological testing for $H$. pylori IgG-antibodies was performed using a commercially available enzyme-linked immunosorbent assay (ELISA) kit (Pyloriset $^{\circledR}$ new EIA-G, Orion Diagnostica, Espoo, Finland). Results were considered positive if the antibody titers were $\geq 250$ International Units per $\mathrm{mL}(\mathrm{IU} / \mathrm{mL})$, according to the manufacturer's guidelines. This assay has been assessed, in a population similar to the population in the presented trial, and has proven a sensitivity and specificity in the Netherlands of $98-100 \%$ and $79-85 \%$, even in patients on acid suppressive therapy $[11,30,31]$.

\section{Culture}

Biopsy specimens of corpus and antrum taken during endoscopy were inoculated onto Columbia agar (Becton Dickinson, Cockeysville, MD, USA) with 10\% lysed horse blood (Bio Trading, Mijdrecht, The Netherlands), and onto Columbia agar with $H$. pylori selective supplement (Oxoid, Basingstoke, UK). Media were then incubated for 72 hours at $37^{\circ} \mathrm{C}$ under microaerophilic conditions $\left(5 \% \mathrm{O}_{2}, 10 \% \mathrm{CO}_{2}\right.$ and $\left.85 \% \mathrm{~N}_{2}\right)$. The isolated colonies of H. pylori were identified by Gram stain showing spiralshaped Gram-negative rods, producing urease rapidly, with positive catalase and oxidase tests.

\section{Histology}

Biopsy specimens were stained for Hematoxylin and Eosin (H\&E) according to the standard procedure. For immunohistochemical (IHC) staining, the slides were heated in an autoclave (Kavoklave, Prestige Medical Ltd, $\mathrm{UK}$ ) in a citric-acid solution ( $\mathrm{pH}=6$ to $121-126^{\circ} \mathrm{C}$ during 30 minutes for antigen retrieval. The slides were then incubated in a Shandon Sequenza Immunostaining Center (Thermo Electron Corporation, the Netherlands) with a polyclonal rabbit IgG anti-Helicobacter pylori antibody (DakoCytomation, Denmark, dilution 1:300), followed by biotinylated goat anti-polyvalent antibody (LabVision Corporation, USA), strepavidin peroxidase (LabVision Corporation, USA) and Liquid DAB + substrate chromogen system (DakoCytomation, Denmark), and counterstained with hematoxylin.

All stained biopsy specimens of corpus and antrum taken during endoscopy were examined by a single expert pathologist who was blinded for clinical data, treatment allocation and other test results.

\section{Gold standard definition}

As the gold standard for H. pylori infection in this study, at 3 months a patient was defined as being $H$. pylori positive on the basis of a positive culture for $H$. pylori or, in the case of a negative culture, a positive examination of either H\&E or IHC stains in combination with H. pylori IgG-antibody titers persistently $\geq 250 \mathrm{IU} / \mathrm{mL}$. At 12 months, a patient was defined as being $H$. pylori positive on the basis of a positive culture for $H$. pylori or, in the case of a negative culture, a positive examination of either H\&E or IHC stains in biopsy samples at 3 months in combination with $H$. pylori IgG-antibody titers persistently $\geq 250 \mathrm{IU} / \mathrm{mL}$ at 12 months.

\section{Statistical analysis}

Continuous variables with a normal distribution were expressed as mean with standard deviation (SD), and continuous variables with a non-normal distribution as median with interquartile range (IQR). Differences between groups were analysed using Students t-test, Mann-Whitney U test, Pearson's Chi-square test or Fisher's Exact test in case of low expected values. For all analyses $P<0.05$, two sided, was considered significant. All analyses were performed with SPSS for Windows, version 19.0 (SPSS, Chicago, IL, USA). Receiver Operating Characteristic (ROC) curves and likelihood ratios were analysed with MedCalc for windows, version 12.1.3.0. Differences in the proportions of patients were analyzed with $95 \%$ confidence interval using the Confidence Interval Analysis software for Windows (version 2.2.0).

\section{Results}

A total of 347 patients were included in the present study. The treatment groups (172 patients in the eradication group and 175 patients receiving placebo) were similar in terms of demographics, rheumatic disease, NSAIDs and other drug use. Our eligibility criteria resulted in a study group with mainly inflammatory rheumatic diseases (rheumatoid arthritis 61\%, spondyloarthropathy $8 \%$, psoriatic arthritis $7 \%$, osteoarthritis $9 \%$, other $15 \%)$. The most commonly used NSAIDs were 
diclofenac (29\%), naproxen (18\%), and ibuprofen (13\%). The mean age was 60 years (SD 10), 61\% was female. Twenty-two patients had a known allergy for amoxicillin and received metronidazole instead (10 patients) or placebo (12 patients). Forty-eight percent used a gastroprotective drug (7\% $\mathrm{H} 2$ receptor antagonists (H2RA), 37\% proton pump inhibitors (PPI), 7\% misoprostol, 3\% used a combination of these).

At baseline, Anti- $H$. pylori IgG antibodies were present in all 347 patients (median titre 1689 (IQR 700-3732). At three months, data on both culture and histology were available in 305 patients; 152 in the eradication group and 153 in the placebo group. In two cases only culture data were available and in 1 case only histology was available. All three cases met the criteria for $H$. pylori-positivity and were found in the placebo group. A total of 32 patients (with no significant differences between eradication and placebo groups) refused the 3month endoscopy, withdrew informed consent, or could not undergo endoscopy because of adverse events. Seven patients used anticoagulant therapy, ruling out biopsy sampling in accordance with the study protocol, and in one patient no biopsy specimens could be obtained because of discomfort requiring early completion of the procedure.

The results of $H$. pylori detection by each of the different tests are shown in Table 1 . Out of the 152 patients who had been treated with $H$. pylori eradication therapy, 141 (93\%) had a negative culture, and of the 153 patients who had been receiving placebo, $54(35 \%)$ had a negative culture $(P<0.001)$. Out of the 152 patients who had been treated with $H$. pylori eradication therapy, 133 (88\%) had a negative H\&E stain, compared to 41 (27\%) of the 153 patients who had been receiving placebo $(P<0.001)$. In the subgroup (with statistically similar

Table 1 Results of $H$. pylori detection by each test

\begin{tabular}{llcc}
\hline Test & & Positives (\%) & Negatives (\%) \\
\hline $\boldsymbol{T}=\mathbf{3 m o n t h s}$ & & \\
Culture (N=305) & Eradication & $11(7)$ & $141(93)$ \\
& Placebo & $99(65)$ & $54(35)$ \\
H\&E stains (N=305) & Eradication & $19(12)$ & $133(88)$ \\
& Placebo & $112(73)$ & $41(27)$ \\
IHC stains (N=68) & Eradication & $29(85)$ & $5(15)$ \\
& Placebo & $7(20)$ & $27(79)$ \\
Serology (N=203) & Eradication & $92(91)$ & $9(9)$ \\
& Placebo & $94(92)$ & $8(8)$ \\
T=12months & & & \\
Serology (N=304) & Eradication & $96(64)$ & $55(36)$ \\
& Placebo & $138(90)$ & $15(10)$ \\
\hline
\end{tabular}

H\&E: hematoxylin and eosin, IHC: immunohistochemistry. Positive serology was defined as $H$. pylori lgG-antibody titers $\geq 250 \mathrm{IU} / \mathrm{mL}$. baseline characteristics as the whole population, data not shown) of 68 patients in which IHC stains were performed, 29 (85\%) of the 34 patients who had been treated with $H$. pylori eradication therapy had a negative IHC stain, compared to 7 (21\%) of the 34 patients in the placebo group $(P<0.001)$. There were no differences between patients using gastroprotection compared to patients who did not take gastroprotective drugs for the presence of $H$. pylori by culture or histology ( $\mathrm{p}=0.454)$.

According to the gold standard criteria, a patient could be either $H$. pylori positive or $H$. pylori negative. The sensitivity, specificity, positive predictive values (PPV) and negative predictive values (NPV) of each test were calculated for the whole group and also differentiated for preceding $H$. pylori eradication therapy or placebo, as is shown in Table 2. For the combined analysis of $\mathrm{H} \& \mathrm{E}$ and IHC stains, results were positive if either test was positive or results were negative if both tests were negative. According to the gold standard criteria for $H$. pylori infection, $H$. pylori eradication was successful in $133(89.9 \%)$ of the 148 patients who had been treated with $H$. pylori eradication therapy, while 120 (78.9\%) of the 152 patients who had been receiving placebo remained $H$. pylori positive. Gold standard criteria could not be calculated in 4 patients in the eradication group en 1 in the placebo group because of missing or negative

Table 2 Results of the sensitivity, specificity, positive predictive value (PPV) and negative predictive value (NPV) of each test; for the total study group and differentiated for preceding $H$. pylori eradication therapy or placebo

\begin{tabular}{lllll}
\hline Test & Sensitivity (\%) & Specificity (\%) & PPV (\%) & NPV (\%) \\
\hline Culture & & & & \\
Total & 82 & 100 & 100 & 87 \\
Eradication & 73 & 100 & 100 & 97 \\
Placebo & 83 & 100 & 100 & 62 \\
H\&E stains & & & & \\
Total & 93 & 99 & 99 & 94 \\
Eradication & 93 & 99 & 93 & 99 \\
Placebo & 92 & 100 & 100 & 78 \\
Subgroup of 68 patients & & & \\
IHC stains & & & & \\
Total & 100 & 95 & 94 & 100 \\
Eradication & 100 & 94 & 60 & 100 \\
Placebo & 100 & 100 & 100 & 100 \\
H\&E+IHC & & & & \\
Total & 100 & 92 & 91 & 100 \\
Eradication & 100 & 90 & 50 & 100 \\
Placebo & 100 & 100 & 100 & 100 \\
\hline
\end{tabular}

H\&E: hematoxylin and eosin, IHC: immunohistochemistry. 
culture results, or missing serology data in combination with available histology results.

\section{Serology}

At baseline, $H$. pylori IgG-antibody titers varied from $250 \mathrm{IU} / \mathrm{mL}$ to $19029 \mathrm{IU} / \mathrm{mL}$ with a median of $1689 \mathrm{IU} /$ $\mathrm{mL}$ (interquartile range (IQR) 700 to $3732 \mathrm{IU} / \mathrm{mL}$ ) with no significant differences in titers between the groups assigned to $H$. pylori eradication therapy or to placebo $(\mathrm{P}=0.39)$. At endoscopy at 3 months, H. pylori IgGantibody titers varied from $126 \mathrm{IU} / \mathrm{mL}$ to $12800 \mathrm{IU} / \mathrm{mL}$, with a median of $1190 \mathrm{IU} / \mathrm{mL}$ (IQR 500 to $2820 \mathrm{IU} / \mathrm{mL}$ ). Patients who had been treated with $H$. pylori eradication therapy had lower $H$. pylori IgG-antibody titers than those treated with placebo; eradication group $(n=101)$ median $730 \mathrm{IU} / \mathrm{mL}$ (IQR 415 to $1461 \mathrm{IU} / \mathrm{mL}$ ) and placebo group $(\mathrm{n}=102)$ median $2026 \mathrm{IU} / \mathrm{mL}$ (IQR 700 to $3571 \mathrm{IU} / \mathrm{mL}$ ) (median difference $-907,95 \% \mathrm{CI}-1356$ to $-460, \mathrm{P}<0.001$ Figure 1). At serological testing at 12 months, patients who had been treated with $H$. pylori eradication therapy had lower $H$. pylori IgG-antibody titers than those treated with placebo; eradication group ( $\mathrm{n}=151$ ) median $370 \mathrm{IU} / \mathrm{mL}$ (IQR 200 to $672 \mathrm{IU} / \mathrm{mL}$ ) and placebo group $(\mathrm{n}=153)$ median $1340 \mathrm{IU} / \mathrm{mL}$ (IQR 490 to $3272 \mathrm{IU} / \mathrm{mL}$ ) (median difference $-778,95 \% \mathrm{CI}$ -1128 to $-466, \mathrm{P}<0.001$ Figure 1 ).

At 3 months, $H$. pylori IgG-antibody titers had dropped below the $250 \mathrm{IU} / \mathrm{mL}$ threshold for positivity in $17 / 203$ (8.4\%) patients; $9 / 101$ (9\%) in the eradication group and $8 / 102(8 \%)$ in the placebo group $(P=0.78)$. At 12 months, H. pylori IgG-antibody titers had dropped

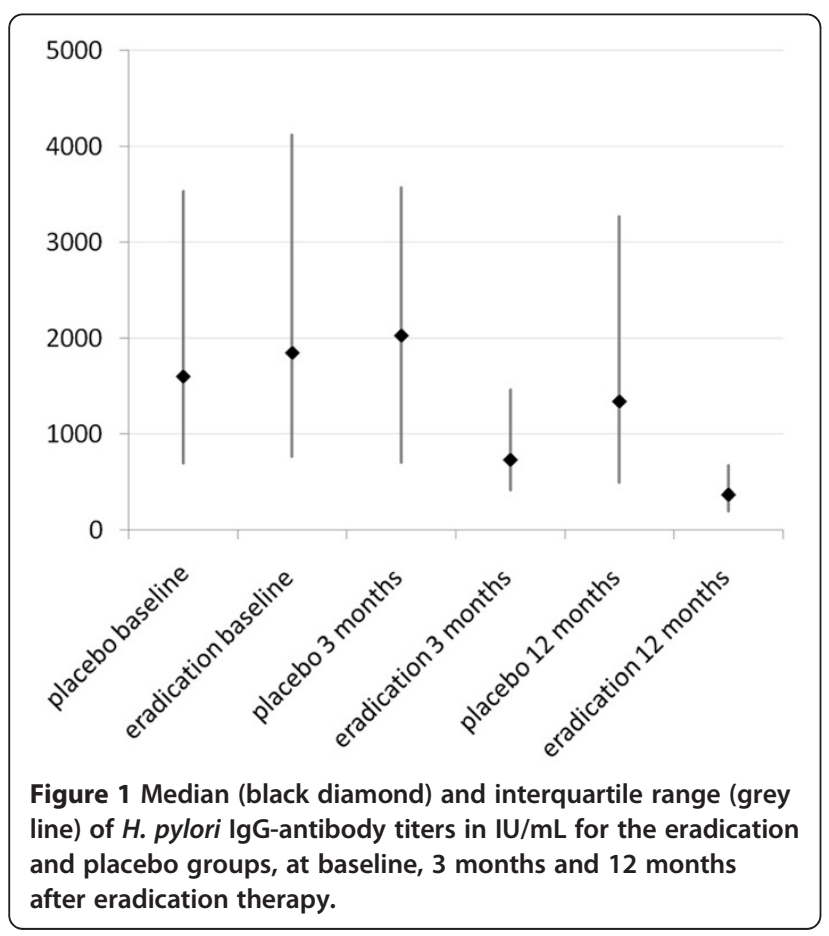

below the $250 \mathrm{IU} / \mathrm{mL}$ threshold for positivity in 70/304 (23\%) patients; $55 / 151$ (36\%) in the eradication group and $15 / 153(10 \%)$ in the placebo group $(P<0.05)$, Table 1.

The absolute change in $H$. pylori IgG-antibody titers from baseline to 3 months (titer at baseline minus titer at 3 months) did differ significantly between the groups; eradication group median change $980 \mathrm{IU} / \mathrm{mL}$ (IQR 190 to $2720 \mathrm{IU} / \mathrm{mL}$ ) and placebo group median change $-26 \mathrm{IU} / \mathrm{mL}$ (IQR -605 (elevation of titer) to $870 \mathrm{IU} / \mathrm{mL}$ ) (median difference 1006, 95\% CI 654 to $1471, P<0.001$ ). The change in $H$. pylori IgG-antibody titers from baseline to 12 months also differed significantly between the groups; eradication group median change $1010 \mathrm{IU} / \mathrm{mL}$ (IQR 363 to $2917 \mathrm{IU} / \mathrm{mL}$ ) and placebo group median change $167 \mathrm{IU} / \mathrm{mL}$ (IQR -337 (elevation of titer) to $1625 \mathrm{IU} / \mathrm{mL}$ ) (median difference 913, 95\% CI 547 to 1362, $P<0.001$ ).

Compared to baseline, at 3 months $H$. pylori IgGantibody titers were median 55\% lower (IQR $24 \%$ to $72 \%$ ) in the eradication group and median $0.9 \%$ lower (IQR $-32 \%$ to $40 \%$ ) in the placebo group (median difference $46 \%, 95 \%$ CI $34 \%$ to $60 \%, P<0.001$ ). Compared to baseline, at 12 months $H$. pylori IgG-antibody titers were median $77 \%$ lower (IQR 48\% to 88\%) in the eradication group and median $22 \%$ lower (IQR $-34 \%$ to $56 \%$ ) in the placebo group (median difference $46 \%, 95 \%$ CI 36 to 58 , $P<0.001)$.

Using the predefined $H$. pylori IgG-antibody titer cutoff point of $\geq 250 \mathrm{IU} / \mathrm{mL}$, serological testing for $H$. pylori IgG-antibodies at endoscopy at 3 months was found to be highly sensitive (99\%) but with very poor specificity (15\%), especially following $H$. pylori eradication therapy (10\%). Arguably, the absolute or percent change in $H$. pylori IgG-antibody titers from baseline represent better methods for evaluating success of $H$. pylori eradication. Figure 2 presents the Receiver Operating Characteristic (ROC) curves for absolute and percent change in $\mathrm{H}$. pylori IgG-antibody titers after 3 and 12 months, associated with a negative result for the gold standard criteria for H. pylori infection. Percent change scores had better diagnostic power in identifying $H$. pylori negative patients at both 3 and 12 months, with area under the ROC curves (AUCs) of 0.62 (95\% CI 0.52 to 0.72, $P=0.343$ ) for absolute change and 0.70 (95\% CI 0.59 to $0.79, P=0.085)$ for percent change at 3 months and 0.73 (95\% CI 0.65 to $0.80, P=0.0016$ ) for absolute change and 0.83 (95\% CI 0.76 to $0.89, P<0.0001$ ) for percent change at 12 months. The optimal cut-off point at 3 months for percent change in H. pylori IgG-antibody titers was $21 \%$, corresponding to a sensitivity of $64 \%$ (95\% CI $31 \%$ to $89 \%$ ) and specificity of $81 \%$ (95\% CI $71 \%$ to $89 \%$ ), negative Likelihood ratio 0.45 (95\% CI 0.2 to 1.1), positive Likelihood ratio 3.3 (95\% CI 2.1 to 5.2 ). 


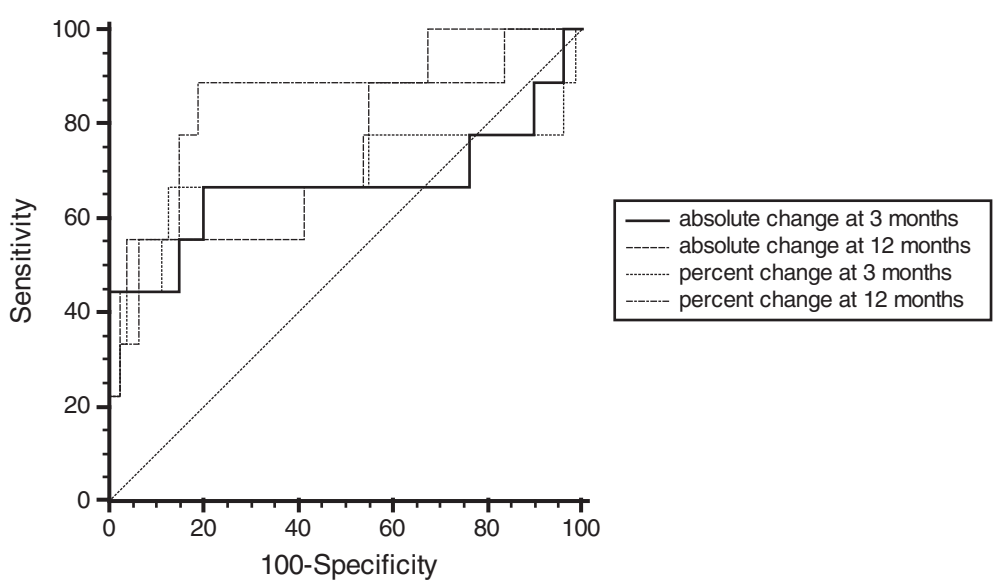

Figure 2 Comparison of ROC curves for absolute and percent change of $H$. pylori-lgG antibody titers at 3 and 12 months after eradication therapy.

The optimal cut-off point at 12 months for percent change in $H$. pylori IgG-antibody titers was $58 \%$, corresponding to a sensitivity of $87 \%$ (95\% CI $60 \%$ to $98 \%$ ) and specificity of $74 \%$ (95\% CI $65 \%$ to $81 \%$ ), negative Likelihood ratio 0.18 ( $95 \%$ CI 0.05 to 0.7 ), positive Likelihood ratio 3.3 (95\% CI 2.6 to 4.1 ).

\section{Discussion}

Following H. pylori eradication therapy or placebo, histological examination of gastric mucosal tissue biopsies provided good sensitivity and specificity ratios for evaluating success of $H$. pylori eradication therapy. In the subgroup with both IHC and H\&E staining, IHC was slightly superior to H\&E. Following eradication therapy both staining methods provided $100 \%$ sensitivity and also very high specificity. A combined analysis of $\mathrm{H} \& \mathrm{E}$ and IHC stains, in which results were positive if either test was positive or results were negative if both tests were negative, did not improve sensitivity while the number of false positive test results increased. Culture of H. pylori in gastric biopsy specimens has very high specificity but relatively low sensitivity [5,32]. In the present study, culture provided $100 \%$ specificity and $82 \%$ sensitivity. However, after $H$. pylori eradication therapy sensitivity dropped to $73 \%$ due to an increasing percentage of false negative cultures. Culture of $H$. pylori therefore does not appear to be very useful for evaluating success of $H$. pylori eradication therapy. In clinical practice, invasive tests for confirmation of eradication should only be used in cases where repeat endoscopy is indicated, for example in patients with gastric ulcer. In all other cases non-invasive test should be employed for follow-up after H. pylori eradication treatment [33].

The choice of a gold standard affects test results of all other tests. According to the guidelines for clinical trials in H. pylori infection, a reliable gold standard should consist of at least 2 methods based on different principles for detecting $H$. pylori infection [5,34]. In the present study, a patient was also considered $H$. pylori positive if culture alone was positive, in view of its absolute specificity. The gold standard in the present study corresponds to acceptable criteria.

Other accurate and relatively inexpensive non-invasive tests that may also be considered for the evaluation of success of $H$. pylori eradication therapy are serology, 13C-urea breath tests and stool antigen tests [33]. While the 13C-urea breath test may have better accuracy (>90\%), the serology test used in this study was less expensive and readily available in all study centres [35]. At the time of the study, stool antigen tests were not yet widely available in the Netherlands. PPI usage (in this study $48 \%$ of the population) may result in false negative test results in both invasive and non-invasive tests, such as culture, histology and 13C-urea breath testing, and should therefore be stopped two weeks before testing [36]. This does not apply for serological testing. Besides, stopping PPI in a population of chronic NSAID users would be non-ethical in a trial setting.

This study shows that in NSAID users, percent change in H. pylori IgG-antibody titers has better diagnostic power in identifying $H$. pylori negative patients at both 3 and 12 months than absolute change in H. pylori IgGantibody titers. Repeated serological testing using a cutoff point of $21 \%$ decrease in $H$. pylori IgG-antibody titers after 3 months and 58\% after 12 months has sufficiently high sensitivity and specificity to be useful for evaluating the success of $H$. pylori eradication therapy. Other groups have found high sensitivity and specificity ratios for percent decrease in $H$. pylori IgG-antibody titers using cut-off points of $25 \%$ at 6 months and $40 \%$ at 3 to 6 months $[10,11,37]$. Using a predefined H. pylori IgGantibody titer cut-off point of $250 \mathrm{IU} / \mathrm{mL}$, repeated 
serological testing for $H$. pylori IgG-antibodies was found to have little diagnostic value.

Overall, NSAID use did not seem to influence $H$. pylori eradication rates or serological testing for $H$. pylori IgG-antibodies, when compared to other studies with patients who do not take NSAIDs [5,32]. Although studies on $H$. pylori IgG serology are not new, there is some data available in which has been shown that NSAID treatment significantly decreases the degree of gastric inflammation [22-25]. However in some studies aspirin and NSAID possibly suppresses the growth of $H$. pylori and may influence diagnostic testing and increase its susceptibility to the antibiotics [38-40]. It is therefore possible that in patients with $H$. pylori infection, concurrent NSAID treatment may affect levels of gastric inflammation and may consequently affect the serological response. While several studies have investigated the time course of $H$. pylori antibody titers after $H$. pylori eradication therapy, none have been conducted in NSAID users yet. Theoretically, if NSAID treatment decreases the degree of gastric inflammation and subsequently affects the serological response, one would not expect to find many false positive test results. However, such an effect still cannot be ruled out because in the present study, a relatively strong decline in $H$. pylori IgG-antibodies was noted 3 months after $H$. pylori eradication (median 55\% decline at 3 months and median $77 \%$ decline at 12 months), compared to other studies. A previous longitudinal analysis of $H$. pylori IgG-antibody titers following successful $H$. pylori eradication demonstrated a mean decline of $26 \%$ at 3 months, $43 \%$ at 6 months, and 55\% at nine months follow-up, after which titers appeared to plateau at approximately 50\% compared to baseline [28].

\section{Conclusions}

In the present study in NSAID taking patients, following H. pylori eradication therapy or placebo, histological examination of gastric mucosal tissue biopsies provided good sensitivity and specificity ratios. The H\&E and IHC staining methods provided comparable high sensitivity and specificity but combining IHC and H\&E did not improve results. A percentual $H$. pylori IgG-antibody titer change has better sensitivity and specificity than an absolute titer change or a predefined H. pylori IgGantibody titer cut-off point for evaluating success of $H$. pylori eradication therapy.

\section{Competing interests}

The author(s) declare that they have no competing interests.

\section{Authors' contributions}

HEV carried out analyses and drafted the manuscript. HDL participated in the design of the study and coordination, carried out the analyses and drafted the manuscript. MVL conceived of the study, and participated in its design and coordination and helped to draft the manuscript. JVB carried out pathological assessments. KSS participated in the design of the study and helped to draft the manuscript. WFL conceived of the study, and participated in its design and coordination and helped to draft the manuscript. JWB conceived of the study, and participated in its design and coordination and helped to draft the manuscript. EJK conceived of the study, and participated in its design and coordination and helped to draft the manuscript. $\mathrm{HMH}$ participated in the design of the study and helped to draft the manuscript. MJ participated in the design of the study and helped to draft the manuscript. BAD conceived of the study, and participated in its design and coordination and helped to draft the manuscript. All authors read and approved the final manuscript.

\section{Acknowledgements}

Funded by: Health Care Insurance Board, the Netherlands; Grant Number: OG-98-22.

\section{Author details}

${ }^{1}$ Arthritis Center Twente, Department of Rheumatology and Clinical Immunology, Medisch Spectrum Twente Hospital and University of Twente, P.O. Box 50.000, 7500 KA Enschede, The Netherlands. ${ }^{2}$ Department of Rheumatology, VU University Medical Center and Jan van Breemen Institute, Amsterdam, The Netherlands. ' ${ }^{3}$ aboratorium Pathologie Oost-Nederland, Enschede, The Netherlands. ${ }^{4}$ Department of Rheumatology and Clinical Immunology, University Medical Center Utrecht, Utrecht, The Netherlands. ${ }^{5}$ Department of Gastroenterology and Hepatology, Erasmus MC University Medical Center, Rotterdam, The Netherlands. 'Department of Rheumatology, Atrium Medical Center, Heerlen, The Netherlands. ${ }^{7}$ Department of

Rheumatology, Rijnstate Hospital, Arnhem, The Netherlands.

Received: 9 June 2012 Accepted: 19 September 2012

Published: 24 September 2012

\section{References}

1. Marshall BJ, Warren JR: Unidentified curved bacilli in the stomach of patients with gastritis and peptic ulceration. Lancet 1984, 1:1311-1315.

2. Rauws EA, Tytgat GN: Cure of duodenal ulcer associated with eradication of Helicobacter pylori. Lancet 1990, 335:1233-1235.

3. Montalban C, Manzanal A, Boixeda D, Redondo C, Bellas C: Treatment of low-grade gastric MALT lymphoma with Helicobacter pylori eradication. Lancet 1995, 345:798-799.

4. Parsonnet J, Friedman GD, Vandersteen DP, Chang Y, Vogelman JH, Orentreich N, Sibley RK: Helicobacter pylori infection and the risk of gastric carcinoma. N Engl J Med 1991, 325:1127-1131.

5. Kullavanijaya $P$, Thong-Ngam D, Hanvivatvong O, Nunthapisud $P$, Tangkijvanich P, Suwanagool P: Analysis of eight different methods for the detection of Helicobacter pylori infection in patients with dyspepsia. J Gastroenterol Hepatol 2004, 19:1392-1396.

6. Talley NJ, Newell DG, Ormand JE, Carpenter HA, Wilson WR, Zinsmeister AR, Perez-Perez Gl, Blaser MJ: Serodiagnosis of Helicobacter pylori: comparison of enzyme-linked immunosorbent assays. J Clin Microbiol 1991, 29:1635-1639.

7. Oksanen A, Veijola L, Sipponen P, Schauman KO, Rautelin H: Evaluation of Pyloriset Screen, a rapid whole-blood diagnostic test for Helicobacter pylori infection. J Clin Microbiol 1998, 36:955-957.

8. Perez-Perez Gl, Dworkin BM, Chodos JE, Blaser MJ: Campylobacter pylori antibodies in humans. Ann Intern Med 1988, 109:11-17.

9. Lahner E, Bordi C, Di GE, Caruana P, D'Ambra G, Milione M, Grossi C, Delle FG, Annibale B: Role of Helicobacter pylori serology in atrophic body gastritis after eradication treatment. Aliment Pharmacol Ther 2002, 16:507-514.

10. Bergey B, Marchildon P, Peacock J, Megraud F: What is the role of serology in assessing Helicobacter pylori eradication? Aliment Pharmacol Ther 2003, 18:635-639.

11. Lerang F, Moum B, Mowinckel P, Haug JB, Ragnhildstveit E, Berge T, Bjorneklett A: Accuracy of seven different tests for the diagnosis of Helicobacter pylori infection and the impact of $\mathrm{H} 2$-receptor antagonists on test results. Scand J Gastroenterol 1998, 33:364-369.

12. Bermejo F, Boixeda D, Gisbert JP, Sanz JM, Canton R, Defarges V, Martin-de-Argila C: Concordance between noninvasive tests in detecting Helicobacter pylori and potential use of serology for monitoring eradication in gastric ulcer. J Clin Gastroenterol 2000, 31:137-141. 
13. Kawai T, Kawakami K, Kudo T, Ogiahara S, Handa Y, Moriyasu F: A new serum antibody test kit (E plate) for evaluation of Helicobacter pylori eradication. Intern Med 2002, 41:780-783.

14. Fabre R, Sobhani I, Laurent-Puig P, Hedef N, Yazigi N, Vissuzaine C, Rodde Potet $F$, Mignon M, Etienne JP, et al: Polymerase chain reaction assay for the detection of Helicobacter pylori in gastric biopsy specimens: comparison with culture, rapid urease test, and histopathological tests. Gut 1994, 35:905-908.

15. Marzio L, Angelucci D, Grossi L, Diodoro MG, Di CE, Cellini L: AntiHelicobacter pylori specific antibody immunohistochemistry improves the diagnostic accuracy of Helicobacter pylori in biopsy specimen from patients treated with triple therapy. Am J Gastroenterol 1998, 93:223-226.

16. Huang $\mathrm{JQ}$, Sridhar $\mathrm{S}$, Hunt $\mathrm{RH}$ : Role of Helicobacter pylori infection and non-steroidal anti- inflammatory drugs in peptic-ulcer disease: a metaanalysis. Lancet 2002, 359:14-22.

17. Kamada T, Hata J, Kusunoki H, Sugiu K, Tanimoto T, Mihara M, Hamada H, Kido S, Dongmei Q, Haruma K: Endoscopic characteristics and Helicobacter pylori infection in NSAID-associated gastric ulcer. $J$ Gastroenterol Hepatol 2006, 21:98-102.

18. Chan FK, Sung JJ, Chung SC, To KF, Yung MY, Leung VK, Lee YT, Chan CS, Li EK, Woo J: Randomised trial of eradication of Helicobacter pylori before non- steroidal anti-inflammatory drug therapy to prevent peptic ulcers. Lancet 1997, 350:975-979.

19. Chan FK, To KF, Wu JC, Yung MY, Leung WK, Kwok T, Hui Y, Chan HL, Chan CS, Hui $E$, et al: Eradication of Helicobacter pylori and risk of peptic ulcers in patients starting long-term treatment with non-steroidal antiinflammatory drugs: a randomised trial. Lancet 2002, 359:9-13.

20. Hawkey CJ, Tulassay Z, Szczepanski L, van Rensburg CJ, FilipowiczSosnowska A, Lanas A, Wason CM, Peacock RA, Gillon KR: Randomised controlled trial of Helicobacter pylori eradication in patients on nonsteroidal anti-inflammatory drugs: HELP NSAIDs study. Helicobacter Eradication for Lesion Prevention. Lancet 1998, 352:1016-1021.

21. de Leest HT, Steen KS, Lems WF, Bijlsma JW, Van de Laar MA, Huisman AM, Vonkeman HE, Houben HH, Kadir SW, Kostense PJ, et al: Eradication of Helicobacter pylori does not reduce the incidence of gastroduodenal ulcers in patients on long-term NSAID treatment: double-blind, randomized, placebo-controlled trial. Helicobacter 2007, 12:477-485.

22. Fu S, Ramanujam KS, Wong A, Fantry GT, Drachenberg CB, James SP, Meltzer SJ, Wilson KT: Increased expression and cellular localization of inducible nitric oxide synthase and cyclooxygenase 2 in Helicobacter pylori gastritis. Gastroenterology 1999, 116:1319-1329.

23. Tatsuguchi A, Sakamoto C, Wada K, Akamatsu T, Tsukui T, Miyake K, Futagami S, Kishida T, Fukuda Y, Yamanaka N, et al: Localisation of cyclooxygenase 1 and cyclooxygenase 2 in Helicobacter pylori related gastritis and gastric ulcer tissues in humans. Gut 2000, 46:782-789.

24. Wambura C, Aoyama N, Shirasaka D, Sakai T, Ikemura T, Sakashita M, Maekawa S, Kuroda K, Inoue T, Ebara S, et al: Effect of Helicobacter pyloriinduced cyclooxygenase-2 on gastric epithelial cell kinetics: implication for gastric carcinogenesis. Helicobacter 2002, 7:129-138.

25. Wambura C, Aoyama N, Shirasaka D, Kuroda K, Maekawa S, Ebara S, Watanabe Y, Tamura T, Kasuga M: Influence of gastritis on cyclooxygenase-2 expression before and after eradication of Helicobacter pylori infection. Eur J Gastroenterol Hepatol 2004, 16:969-979.

26. Meyer F, Ramanujam KS, Gobert AP, James SP, Wilson KT: Cutting edge: cyclooxygenase- 2 activation suppresses Th1 polarization in response to Helicobacter pylori. J Immunol 2003, 171:3913-3917.

27. Kim TI, Lee YC, Lee KH, Han JH, Chon CY, Moon YM, Kang JK, Park IS: Effects of nonsteroidal anti-inflammatory drugs on Helicobacter pylori-infected gastric mucosae of mice: apoptosis, cell proliferation, and inflammatory activity. Infect Immun 2001, 69:5056-5063.

28. Cutler AF, Prasad VM, Santogade P: Four-year trends in Helicobacter pylori IgG serology following successful eradication. Am J Med 1998, 105:18-20.

29. de Leest HT, Steen KS, Lems WF, Bijlsma JW, Van de Laar MA, Huisman AM, Vonkeman HE, Houben HH, Kadir SW, Kostense PJ, et al: Eradication of Helicobacter pylori does not reduce the incidence of gastroduodenal ulcers in patients on long-term NSAID treatment: double-blind, randomized, placebo-controlled trial. Helicobacter 2007, 12:477-485.

30. Meijer BC, Thijs JC, Kleibeuker JH, van Zwet AA, Berrelkamp RJ: Evaluation of eight enzyme immunoassays for detection of immunoglobulin $G$ against Helicobacter pylori. J Clin Microbiol 1997, 35:292-294.
31. van de Wouw BA, de Boer WA, Jansz AR, Roymans RT, Staals AP: Comparison of three commercially available enzyme-linked immunosorbent assays and biopsy-dependent diagnosis for detecting Helicobacter pylori infection. J Clin Microbiol 1996, 34:94-97.

32. Kisa O, Albay A, Mas MR, Celasun B, Doganci L: The evaluation of diagnostic methods for the detection of Helicobacter pylori in gastric biopsy specimens. Diagn Microbiol Infect Dis 2002, 43:251-255.

33. Malfertheiner P, Megraud F, O'Morain CA, Atherton J, Axon AT, Bazzoli F, Gensini GF, Gisbert JP, Graham DY, Rokkas T, et al: Management of Helicobacter pylori infection-the Maastricht IV/ Florence Consensus Report. Gut 2012, 61:646-664.

34. Technical annex: tests used to assess Helicobacter pylori infection: Working Party of the European Helicobacter pylori Study Group. Gut 1997, 41(Suppl 2):S10-S18.

35. Lindsetmo RO, Johnsen $\mathrm{R}$, Eide $\mathrm{TJ}$, Gutteberg $\mathrm{T}$, Husum $\mathrm{HH}$, Revhaug A: Accuracy of Helicobacter pylori serology in two peptic ulcer populations and in healthy controls. World J Gastroenterol 2008, 14:5039-5045.

36. Kokkola A, Rautelin H, Puolakkainen P, Sipponen P, Farkkila M, Haapiainen R, Kosunen TU: Diagnosis of Helicobacter pylori infection in patients with atrophic gastritis: comparison of histology, 13C-urea breath test, and serology. Scand J Gastroenterol 2000, 35:138-141.

37. Marchildon $\mathrm{P}$, Balaban $\mathrm{DH}$, Sue $\mathrm{M}$, Charles C, Doobay R, Passaretti $N$, Peacock J, Marshall BJ, Peura DA: Usefulness of serological IgG antibody determinations for confirming eradication of Helicobacter pylori infection. Am J Gastroenterol 1999, 94:2105-2108.

38. Gu Q, Xia HH, Wang WH, Wang JD, Wong WM, Chan AO, Yuen MF, Lam SK, Cheung HK, Liu XG, et al: Effect of cyclo-oxygenase inhibitors on Helicobacter pylori susceptibility to metronidazole and clarithromycin. Aliment Pharmacol Ther 2004, 20:675-681.

39. Park SH, Park DI, Kim SH, Kim HJ, Cho YK, Sung IK, Sohn Cl, Jeon WK, Kim BI, Keum DK: Effect of high-dose aspirin on Helicobacter pylori eradication Dig Dis Sci 2005, 50:626-629.

40. Wang WH, Wong WM, Dailidiene D, Berg DE, Gu Q, Lai KC, Lam SK, Wong $\mathrm{BC}$ : Aspirin inhibits the growth of Helicobacter pylori and enhances its susceptibility to antimicrobial agents. Gut 2003, 52:490-495.

doi:10.1186/1471-230X-12-133

Cite this article as: Vonkeman et al: Assessment of Helicobacter pylori eradication in patients on NSAID treatment. BMC Gastroenterology 2012 $12: 133$.

\section{Submit your next manuscript to BioMed Central and take full advantage of:}

- Convenient online submission

- Thorough peer review

- No space constraints or color figure charges

- Immediate publication on acceptance

- Inclusion in PubMed, CAS, Scopus and Google Scholar

- Research which is freely available for redistribution 